\title{
Frequency and Temperature Dependence of Acoustic Properties of Polymers Used in Pulse-Echo Systems
}

\author{
J. E. Carlson ${ }^{\dagger, *}$, J. van Deventer ${ }^{\dagger}$, A. Scolan ${ }^{\dagger}$, and C. Carlander ${ }^{\ddagger}$ \\ ${ }^{\dagger}$ EISLAB, Dept. of Computer Science and Electrical Engineering, Luleå University of Technology, SE-971 87 Luleå, Sweden. \\ ${ }^{\ddagger}$ D-Flow AB, Aurorum 2, SE-977 75 Luleå, Sweden \\ *Email: Johan.Carlson@sm.luth.se
}

\begin{abstract}
In ultrasonic pulse-echo systems, polymers like PMMA (Polymethylmethacrylate) and PEEK (Polyetheretherketone) are often used as buffer-rods, placed between the ultrasound transducer and the unknown material (liquid, gas, or solid material). Provided the acoustic properties of the buffer-rods are known, it is possible to calculate these also for the unknown material, based on reflections between the buffer-rod and the unknown medium. However, temperature changes also affect these properties.
\end{abstract}

In this paper we present a method for measuring acoustic attenuation, speed of sound and density, for buffer-rod materials. We also give experimental values for PMMA and PEEK, for temperatures between $5^{\circ} \mathrm{C}$ and $37^{\circ} \mathrm{C}$, and for $5 \mathrm{MHz}$ and $10 \mathrm{MHz}$ ultrasound frequency.

\section{INTRODUCTION}

Ultrasonic pulse-echo systems are widely used to estimate properties of liquids and gases. A common principle is to use a buffer material (buffer-rod) fixed to the ultrasound transducer. Assuming the acoustic properties of the buffer-rod are known, it is then possible to calculate the acoustic impedance of the unknown material from reflections between the buffer-rod and the unknown material. From acoustic impedance and speed of sound it is the possible to calculate density and adiabatic bulk modulus of the material. This was first introduced by Lynnworth [1] and Papadakis [2], and later further developed by Püttmer [3] and Deventer [4] for density measurement of liquids.

For some buffer-rod materials, like PMMA values for speed of sound and density are available in the literature. A problem is that these properties depends on both temperature and on ultrasound frequency, and this dependency is normally not documented. For other existing and potential buffer-rod materials, no information about their acoustic properties is available.

In this paper we present a technique to measure acoustic attenuation, speed of sound, and density of polymers. Experimental results are presented for PMMA and PEEK.

0-7803-7922-5/03/\$17.00 (c) 2003 IEEE

\section{THEORY}

To measure acoustic attenuation, speed of sound, and density, we use the configuration shown in Fig. 1. The transducer first transmits a short ultrasound pulse. This pulse then propagates through the water and encounters the boundary (at normal angle) between the water and the polymer sample, where part is reflected, and the rest continues into the polymer sample. At the bottom of the polymer sample, part of the pulse is reflected again. We also record a secondary reflection $\left(x_{3}(t)\right.$ in Fig. 1) that has propagated twice through the polymer samples. The same transducer is then used to record the three echoes $x_{1}(t), x_{2}(t)$, and $x_{3}(t)$, as labeled in Fig. 1.

From the measurement configuration, we then get the following relationships (for continuous wave ultrasound) [5]:

$$
\begin{aligned}
A_{1} & =A_{0} R_{12} e^{-2 d_{1} \alpha_{1}} \\
A_{2} & =A_{0} T_{12} R_{21} T_{21} e^{-2 d_{1} \alpha_{1}} e^{-2 d_{2} \alpha_{2}} \\
A_{3} & =A_{0} T_{12} R_{21}^{3} T_{21} e^{-2 d_{1} \alpha_{1}} e^{-4 d_{2} \alpha_{2}} \\
R_{21} & =-R_{12} \\
T_{12} & =1+R_{12} \\
T_{21} & =1+R_{21}=1-R_{12} \\
R_{12} & =\frac{z_{2}-z_{1}}{z_{2}+z_{1}},
\end{aligned}
$$

where $A_{0}, A_{1}, A_{2}$, and $A_{3}$ are the amplitudes of the transmitted ultrasound pulse, and the reflected echoes $x_{1}(t), x_{2}(t)$, and $x_{3}(t), \alpha_{1}$ and $\alpha_{2}$ are the attenuation coefficients of the water and the polymer, respectively, while $R_{12}, T_{12}, R_{21}$, and $T_{21}$ are the reflection and transmission coefficients from water to polymer and from polymer to water, respectively. The reflection coefficients can be expressed in terms of the specific acoustic impedances of the water, $z_{1}$, and the polymer, $z_{2}$. Since the acoustic impedances $z_{1}$ and $z_{2}$ is given by

$$
\begin{aligned}
& z_{1}=\rho_{1} c_{1} \\
& z_{2}=\rho_{2} c_{2},
\end{aligned}
$$



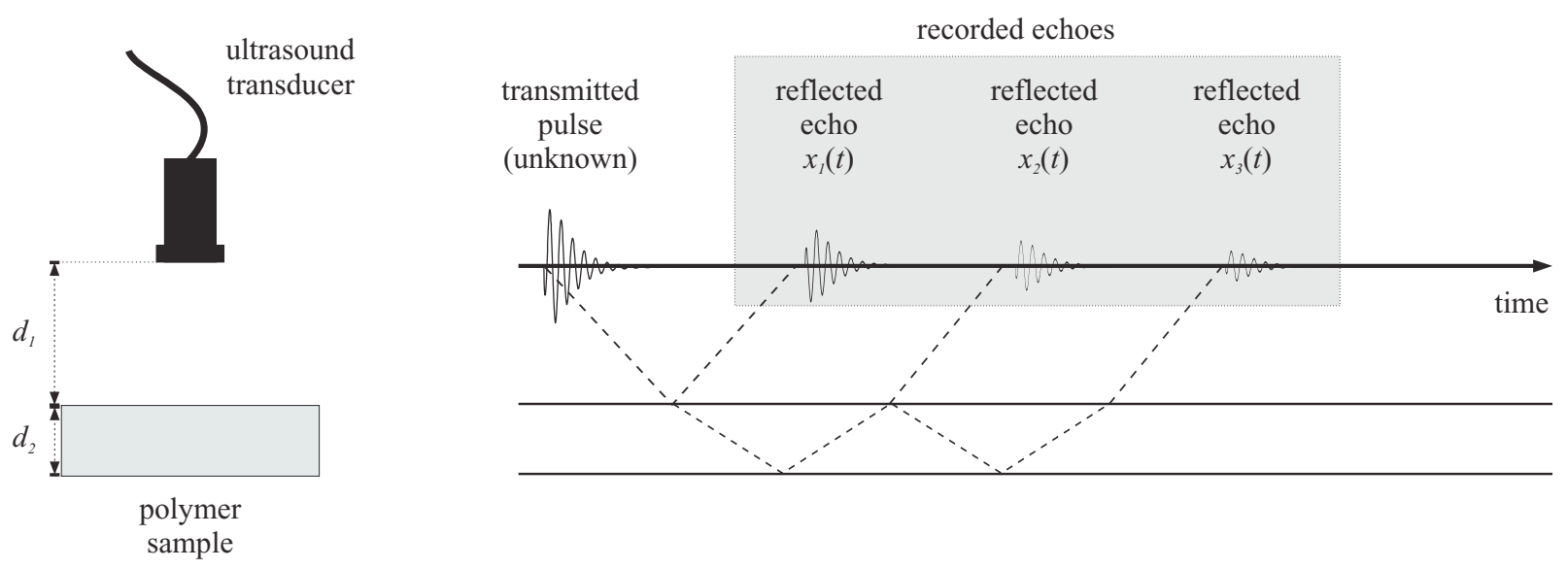

Fig. 1. Working principle of the immersion setup. Primary and secondary reflections from the polymer sample results in three echoes, $x_{1}(t), x_{2}(t)$, and $x_{3}(t)$, as indicated in the figure.

it means that once we know the speed of sound through the polymer $\left(c_{2}\right)$ and its acoustic impedance, $z_{2}$, we also know its density.

Solving the system of equations (1) above for the acoustic impedance, $z_{2}$ and the attenuation coefficient, $\alpha_{2}$ of the polymer sample, yields

$$
\begin{aligned}
\alpha_{2} & =-\frac{1}{2 d_{2}} \ln \left(\frac{A_{1} A_{3}-A_{2}^{2}}{A_{1} A_{2}}\right) \\
z_{2} & =z_{1} \frac{1+R_{12}}{1-R_{12}},
\end{aligned}
$$

where the reflection coefficient $R_{12}$ is given by

$$
R_{12}=\sqrt{\frac{A_{1} A_{3}}{A_{1} A_{3}-A_{2}^{2}}},
$$

the amplitudes are estimated from the reflected echoes, and the acoustic impedance of water is calculated using Eq. (2) and known values for $\rho_{1}[6]$ and $c_{1}[7]$.

\section{A. Estimating Amplitudes}

The amplitudes are estimated from the recorded echoes (see Fig. 1) using the magnitude of the Fourier transform of each of the echoes. In this way we obtain values of the amplitudes as a function of the frequencies present in the pulse. We define the pulse bandwidth, $W$ as the frequency band around each pulse's center frequency, $\omega_{0}$, where the amplitude dropped by $6 \mathrm{~dB}$.

In practice, all calculations are made using sampled versions of the pulses, and using the Fast Fourier Transform (FFT) to determine the relations above. In this case, we need to take into account also the sampling frequency and the number of points used in the FFT. The modifications to the equations are trivial.

In the time domain representation of the pulses, the amplitudes $A_{1}, A_{2}$ and $A_{3}$ can be negative. When using the magnitude of the Fourier transform to estimate this amplitude, the sign is lost. We do, however know from the physics of the experiment, that the first amplitude $A_{1}$ is always negative compared to $A_{2}$ and $A_{3}$.

\section{B. Estimating Speed of Sound}

The speed of sound in the polymer is also determined as a function of frequency, looking at the group delay between the first two echoes, instead of a standard cross-correlation technique.

Let $X_{1}(\omega)$ and $X_{2}(\omega)$ be the Fourier transforms of the first two echoes, $x_{1}(t)$ and $x_{2}(t)$ respectively.

The group delay is defined as

$$
\Phi(\omega)=\frac{\partial}{\partial \omega} \phi(\omega)
$$

where $\phi(\omega)$ is the phase difference between the two echoes, that is

$$
\phi(\omega)=\arg \left(\frac{X_{2}(\omega)}{X_{1}(\omega)}\right),
$$

for $\omega_{0}-W / 2 \leq \omega \leq \omega_{0}+W / 2$.

From the group delay, the speed of sound, $c_{2}(\omega)$ can be calculated, knowing the thickness, $d_{2}$ of the polymer sample

$$
c_{2}(\omega)=2 d_{2} / \Phi(\omega) .
$$

In the presence of noise, however, the numerical differentiation in Eq. 6 becomes unstable. To overcome this problem, we used a more robust group delay estimator. The details of this estimator can be found in [8].

\section{EXPERIMENTS}

\section{A. Setup}

Fig. 2 shows the experimental setup used in this paper. The probe consists of an ultrasound transducer, with either 
$5 \mathrm{MHz}$ or $10 \mathrm{MHz}$ center frequency, manufactured by Panametrics, Inc. The transducer was fixed to the setup, with a gap of 5-10 mm of water between the transducer surface and the polymer sample. The transducer was excited using a Panametrics 5025PR pulser/receiver. The whole setup was immersed in water and put into a temperature controlled chamber (Heraeus Vötsch HT4010). The temperature chamber was set to the requested value and the temperature was then stabilized for twelve hours. Once the temperature had stabilized, 200 pulses were collected using a Nicolet 460 digitizing oscilloscope, sampling at $200 \mathrm{MHz}$ with a vertical resolution of 8 bits. For each pulse, the temperature of the water was measured using a PT100 sensor connected to a Systemteknik Thermolyzer. In the calculations we assume the temperature is the same for both water and polymer.

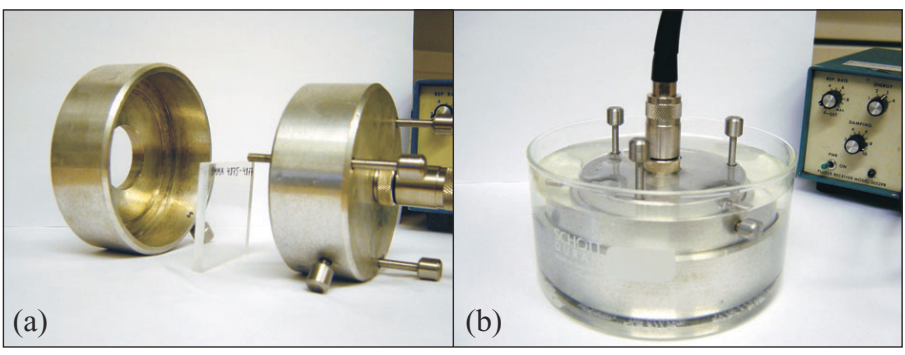

Fig. 2. The immersion setup used in the experiments.

\section{B. Results}

A total of twelve experiments were made, with two different polymers, PMMA and PEEK. For each polymer, experiments were made at $5^{\circ} \mathrm{C}, 20^{\circ} \mathrm{C}$, and $37^{\circ} \mathrm{C}$, using either a $5 \mathrm{MHz}$ or a $10 \mathrm{MHz}$ transducer. All characteristics of the polymers were calculated as a function of frequency, and the Figures 3-5 show this, for the $5 \mathrm{MHz}$ transducer at $20{ }^{\circ} \mathrm{C}$. For the other experiments, average values are shown in Table I. The figures show no confidence interval, since the experimental variation was too small to be visible in the plot. A detailed uncertainty analysis of other error sources will be included in future work. Fig. 3 shows the acoustic attenuation coefficient, $\alpha$ for PMMA and PEEK, for a $5 \mathrm{MHz}$ transducer, at $20^{\circ} \mathrm{C}$. The results agree well with those presented in [4]. Fig. 4 shows that the speed of sound in (measured as the group velocity of the ultrasound pulse) is not constant with frequency. This is expected, but it also means that the pulse shape changes slightly when the pulse passes through the material. If a standard cross-correlation technique is used to estimate speed of sound, the results would be biased [8].

Since density is a physical property of the polymer, it should not depend on which ultrasound frequency we use to measure it. As expected, Fig. 5 shows that the density is constant as a function of frequency.

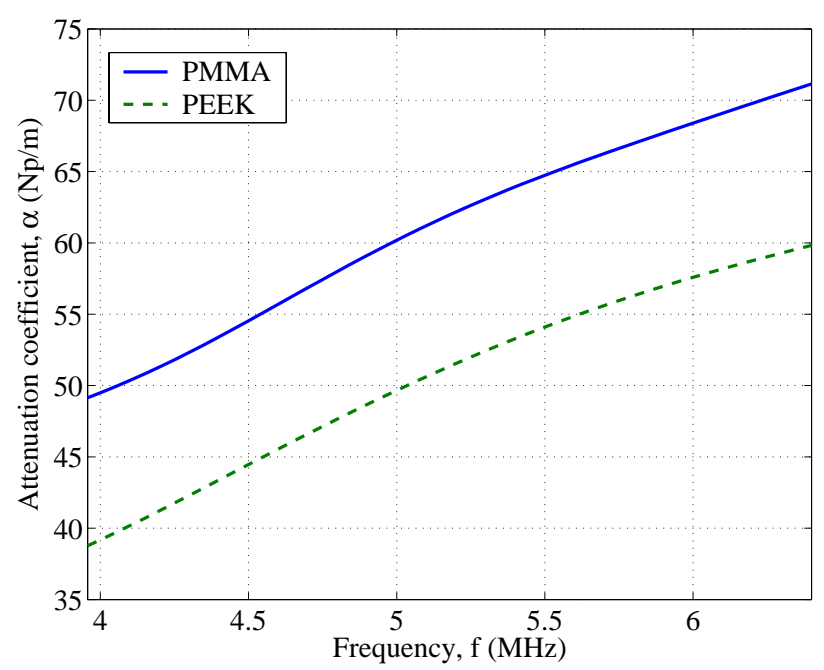

Fig. 3. Acoustic attenuation coefficient as function of ultrasound frequency of PMMA and PEEK at $20{ }^{\circ} \mathrm{C}$, for a transducer with $5 \mathrm{MHz}$ center frequency.

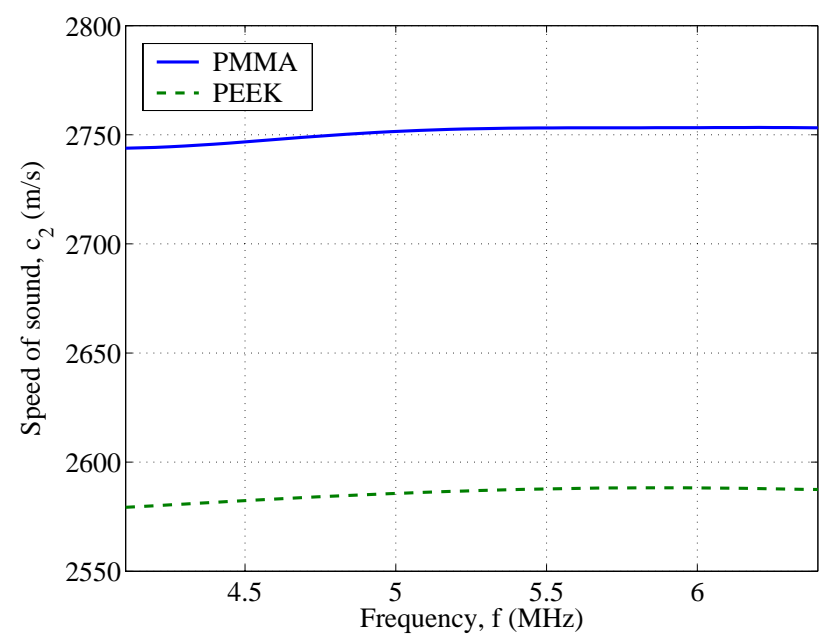

Fig. 4. Speed of sound in PMMA and PEEK as function of ultrasound frequency at $20^{\circ} \mathrm{C}$, for a transducer with $5 \mathrm{MHz}$ center frequency.

Table I shows the estimated values for the other experiments, but averaged over the bandwidth of the pulse. The temperature values within parentheses are the actual measured temperature during the experiment.

The obtained values of density of PMMA and PEEK at 20 ${ }^{\circ} \mathrm{C}$ agree well with those presented by Deventer et al. [9].

\section{DISCUSSION}

We have shown that the immersion setup shown in Fig. 2 can be used to determine values of acoustic attenuation, speed of sound, and density for PMMA and PEEK polymers. We also made experiments with PVDF (Polyvinylidenefluoride) and POM (Polyoxymethylene), but we were unable to obtain values for these. The reason was that for the 
TABLE I

MEASURED PROPERTIES OF PMMA AND PEEK, AVERAGED OVER THE BANDWIDTH OF THE PULSE.

\begin{tabular}{ccccccc}
\hline Material & $\begin{array}{c}\text { Temperature } \\
\left({ }^{\circ} \mathrm{C}\right)\end{array}$ & $\begin{array}{c}\text { Frequency } \\
(\mathrm{MHz})\end{array}$ & $\begin{array}{c}\text { Acoust. impedance } \\
z(\mathrm{MPa} \cdot \mathrm{s} / \mathrm{m})\end{array}$ & $\begin{array}{c}\text { Attenuation, } \\
\alpha(\mathrm{Np} / \mathrm{m})\end{array}$ & $\begin{array}{c}\text { Speed of sound } \\
c(\mathrm{~m} / \mathrm{s})\end{array}$ & $\begin{array}{c}\text { Density } \\
\rho\left(\mathrm{kg} / \mathrm{m}^{3}\right)\end{array}$ \\
\hline PMMA & $5(5.07)$ & 5 & 3.24 & 55.32 & 2795 & 1161 \\
PMMA & $5(5.02)$ & 10 & 3.14 & 83.28 & 2791 & 1125 \\
PMMA & $20(19.34)$ & 5 & 3.28 & 60.84 & 2750 & 1192 \\
PMMA & $20(21.24)$ & 10 & 3.37 & 105.90 & 2759 & 1222 \\
PMMA & $37(37.09)$ & 5 & 3.44 & 61.25 & 2693 & 1277 \\
PMMA & $37(37.14)$ & 10 & 3.69 & 106.31 & 2699 & 1368 \\
PEEK & $5(5.24)$ & 5 & 3.35 & 53.40 & 2617 & 1280 \\
PEEK & $5(4.93)$ & 10 & 3.32 & 103.32 & 2625 & 1264 \\
PEEK & $20(20.21)$ & 5 & 3.24 & 52.59 & 2586 & 1252 \\
PEEK & $20(21.23)$ & 10 & 3.29 & 85.44 & 2589 & 1272 \\
PEEK & $37(36.49)$ & 5 & 3.32 & 44.35 & 2554 & 1300 \\
PEEK & $37(37.29)$ & 10 & 3.26 & 73.63 & 2558 & 1276 \\
\hline
\end{tabular}

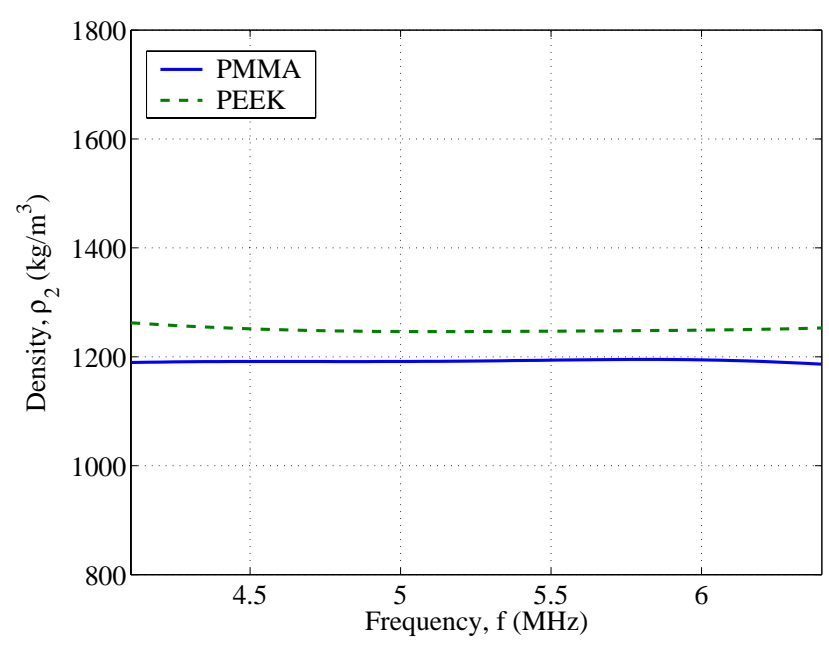

Fig. 5. Density of PMMA and PEEK as function of ultrasound frequency at $20^{\circ} \mathrm{C}$, for a $5 \mathrm{MHz}$ transducer.

frequencies used for PMMA and PEEK, the reflection and attenuation coefficients were too high, and we were able to record at most two echoes. These polymers might, however, be interesting for use with lower ultrasound frequencies.

The main source of error in the present setup, is misalignment of the transducer to the polymer sample. If the surface of the transducer is not parallel to the surface of the sample, we will have an oblique reflection. As a consequence, the attenuation and acoustic impedance over-estimated. This might explain the apparent variation in density between the $5 \mathrm{MHz}$ and the $10 \mathrm{MHz}$ transducers.

Depending on the distance between the transducer and the sample ( $d_{1}$, in Fig. 1), the diffraction losses might also differ between different measurements. At the moment, no correction for diffraction losses is made.

\section{Conclusions}

In this paper, we present a method based on pulse-echo ultrasound to measure acoustic properties of polymers. The experiments show that the proposed techique can be used to obtain values for acoustic attenuation, speed of sound, and density.

These values depend on temperature and ultrasound frequency, and when polymers like PMMA or PEEK are used as buffer-rods, these values have to be known. In this paper we report values for $5{ }^{\circ} \mathrm{C}, 20^{\circ} \mathrm{C}$, and $37^{\circ} \mathrm{C}$, for 5 and 10 $\mathrm{MHz}$ transducers.

\section{REFERENCES}

[1] L. C. Lynnworth, Ultrasonic Measurement for Process Control: Theory, techniques, applications. Boston, MA: Academic Press, 1989.

[2] E. P. Papadakis, K. A. Fowler, and L. C. Lynnworth, "Ultrasonic Attenuation by Spectrum Analysis of Pulses in Buffer Rods: Method and Diffraction Corrections," J. Acoust. Soc. Am., vol. 53, no. 5, pp. 1336-1343, 1973.

[3] A. Püttmer, Ultrasonic Density Sensor for Liquids. PhD thesis, Ottovon-Guericke-Universität Magdeburg, 1998.

[4] J. van Deventer, Material Investigations and Simulation Tools Towards a Design Strategy for an Ultrasonic Densitometer. $\mathrm{PhD}$ thesis, Luleå University of Technology, 2001.

[5] L. E. Kinsler, A. R. Frey, A. B. Coppens, and J. V. Sanders, Fundamentals of Acoustics. New York: Wiley, 3rd ed., 1982.

[6] D. R. Lide, ed., Handbook of Chemistry and Physics. CRC Press, 81st ed., 2001.

[7] N. Bilaniuk and S. K. Wong, "Speed of Sound in Water as a Function of Temperature," J. Acoust. Soc. Am., vol. 93, no. 3, pp. 1609-1612, 1993.

[8] J. E. Carlson, F. Sjöberg, and P.-E. Martinsson, "A noise-tolerant group delay estimator applied to dispersion measurement in gases," in Proc. IEEE Int. Ultrason. Symp., (Honolulu, Hawaii, USA), pp. Paper 3C-2, Oct. 5-8 2003.

[9] J. van Deventer, T. Löfqvist, and J. Delsing, "PSpice modeling of ultrasonic systems," IEEE Trans. on Ultrason., Ferroelec., and Freq. Contr., vol. 47, no. 4, pp. 1014-1024, 2000. 